\title{
Ergonomic Principles to Improve the Use of Cognitive Stimulation Systems for the Elderly: A Comparative Study of Two Software Tools
}

\author{
Gabriel Michel, Eric Brangier, and Mélissa Brun \\ Lorraine University, PErSEUs, UFR SHA, Île du Saulcy, 57006 Metz, France \\ \{Gabriel.Michel, Eric.Brangier\}@univ-lorraine.fr, \\ myla-melissa@hotmail.fr
}

\begin{abstract}
The aim of our communication is to present results of an evaluation of two cognitive stimulation software tools ("ProfessionalTool" and "StudyTool") and to give recommendations to improve their usability. The evaluation was conducted using a test user on a group of 32 seniors (average age 78.19 years) and a group of 15 people (mean age 30.47 years). The "ProfessionalTool" software includes thirty exercises targeting different cognitive skills. The second software - "StudyTool"- has been designed by our team applying user-centered design. The performances of these interfaces were measured using a questionnaire of satisfaction and a heuristic inspection observation grid, based on ergonomic criteria. The scores obtained by each group and each method of data collection were calculated and compared. An important result is that the number of problems encountered by users in the cognitive stimulation tasks is $\mathrm{M}=10.09$ with ProfessionalTool; i.e. a senior user remained stuck for ten minutes on a settings screen. The results of the questionnaire also indicate problems concerning visual ergonomics guidelines, workload, control and error handling, uniformity and consistency, significance and compatibility. This experience highlights the importance of ergonomics in cognitive stimulation software. Their adaptation to a specific public need is often insufficient, especially as the users have troubles with memory and attention. Our study enables us to make a positive contribution of ergonomic human- computer interaction to cognitive stimulation. Beyond the actual effect of cognitive stimulation that is no longer in doubt, the challenge is to support the use and empower the user. This is only possible through tailored interactions.
\end{abstract}

Keywords: Ergonomics, User Experience, Gamification, Persuasive Technology, Emotional Design, Motivation.

\section{Introduction}

Population aging is above all a social and demographic problem. In Europe, the percentage of people aged over 65 years old rose from 7,5\% to 12,5\% between 1950 and 2000 , according to forecasts this percentage could be as high as $30 \%$ by 2050 [6]. 
In France for example, INSEE (the french statistical agency) estimates that the over 65 's will represent $20,1 \%$ of the population by 2020 (13 million) and more than $26 \%$ by 2050 (over 18 million). A third of the population will be over 60 years old by 2050 , compared to one in 5 people in 2000. The proportion of over 60's in the total population will be higher than the under 20's. In other industrialized countries like Japan, Italy, and Germany the phenomenon of population aging is even more critical [1]. Even in China it has been forecast that $23 \%$ of the population will be over 65 by 2050. According to the World Health Organization there will be 2 billion people aged 60 and over by 2050.

As well as the expected labor shortage in the countries hit, this population aging phenomenon will be accompanied by health problems, such as dementia, cognitive impairment, depression, inability to adapt, lack of self efficacy and social isolation, making this population dependent and vulnerable [2]. It will therefore be very valuable to develop efficient mechanisms aiming to reinforce and improve the life functions of the elderly, both for individuals and societies and their efforts to cope with what appears to be a demographic revolution [2].

All existing research shows that being active enables seniors to keep a positive image of themselves, to expand their horizons and to conserve a well being which defies the stereotypes associated with their age.

Among these activities, cognitive stimulation can be the answer to maintaining this well-being by means of regular brain training. These tools, combined with ergonomic knowledge, and specific criterion for this population can provide access to intellectual stimulation using technology which until now has been out of their reach [3].

We will start with a brief overview of cognitive stimulation, then go back to the domain of technology and seniors before presenting our tests and discussing the results.

\section{Cognitive Stimulation and Aging: A Rapidly Expanding Domain}

The current aging of the population has lead to an increase in research programs aiming to find solutions to cognitive impairment and neurodegenerative diseases. For most people cognitive stimulation is still considered as the non drug related management of Alzheimer's disease. But today cognitive stimulation isn't just stimulating cognitive functions such as memory, attention and problem solving through classical exercises. It is a comprehensive educational approach which is cognitive but also psychological and social. It is in fact a concept which is currently expanding in many different directions. The populations targeted are not only elderly people who are ill, but all categories of the population, professionals who use it, applying it to different domains.

The idea is to solicit functions which are less frequently used as people get older. The positive effects of cognitive stimulation in the repetition of the tasks involved are observed. Performance scores vary considerably before and after the training, and closely depend on the basic intellectual level of the participants [4] For people with various types of mental retardation, we will refer to cognitive remediation which we 
observed, enabled a certain amount of progress [5]. Its approach is focused on the reconstruction of the person's inherent abilities, encouraging meta-cognitive processes rather than gaining new knowledge [6].

Previously cognitive stimulation only concerned patients suffering from dementia, but gradually concerned elderly people and people with mental disabilities. Nowadays we hear more and more about the development of CS systems for all categories of the population.

Coming back to seniors, apart from CS, we are already aware of the advantages of using the internet [7]: social interaction, learning, searching for subjects of personal interest, being able to keep up with the latest news, online banking, developing online social networking, online shopping, keeping in contact with friends and family. After the web which is increasingly becoming a part of seniors' lives, CS systems are foreseen as being among the services which will see their use by this population grow rapidly. This will happen as soon as they become more accessible financially and in terms of usability.

\section{Seniors and Technology}

Even today Technology is not accessible to everybody and presents an obstacle for a considerable number of seniors. The complexity of certain interactions, the large amount of information on the same page, the speed this information is presented at, all these features make it difficult for them to search, and even more so for people with mental disabilities [8]. Most of the time, just by simplifying the interface would be enough to noticeably increase user performance. An assessment made of several websites show that usability for seniors was only 2,8\% for text spacing, 5,8\% for guidance and navigation, 9,5\% for audio and video animations, $25 \%$ for hypertext links, and 38\% for the efficacy of the Site Map etc. Even if the tasks were performed successfully, user efficiency, satisfaction and preference levels are not fulfilled [6].

Very few interfaces are adapted to elderly people, even in the domains where universality is essential, for example, the electronic vote [9]. Even if there is a large amount of knowledge which enables seniors to access technology today [10], especially specific ergonomic guidelines, they are not often taken into consideration by the designers. This is the case for cognitive stimulation software programs too.

We initially carried out an ergonomic inspection of several cognitive stimulation software programs. This assessment of the consistency with the guidelines for seniors showed that all the software programs contained errors, proving that the ergonomic guidelines had not been taken into account at the conception phase.

\section{Ergonomic Evaluation}

\subsection{Method}

The evaluation was carried out using a user test, a satisfaction questionnaire and an observation grid. The questionnaire consisted of a data sheet and a satisfaction evaluation, based on Bastien and Scapin criteria [11]. It included 6 categories and 
18 items presented in the form of a 5 point Likert scale. The observation grid accounted for the occurrence of difficulties, participants' remarks, timing of sessions and the number of game requests expressed by our participants.

\subsection{Presentation of the Interfaces}

Two cognitive stimulation exercises were tested. The first one was the « Displaced characters » from the ProfessionalTool software program (a well known software in the field of cognitive stimulation softwares), which aims to boost mental functions, particularly those of seniors. This program appears to be the most popular one used in France, the one most often mentioned in articles about cognitive stimulation and the most common one on the internet. ProfessionalTool consists of thirty exercises targeting different cognitive skills. We chose one of the most typical which is a memory training exercise (see Fig.1).

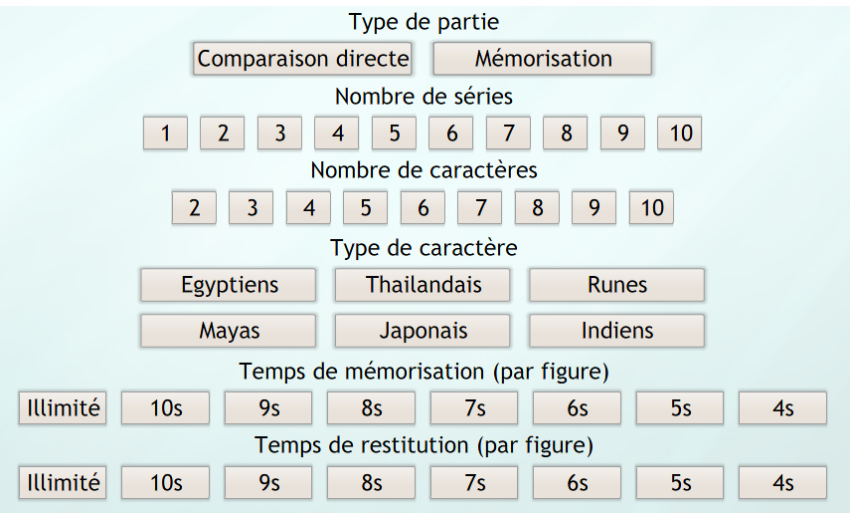

Fig. 1. ProfessionalTool settings screen

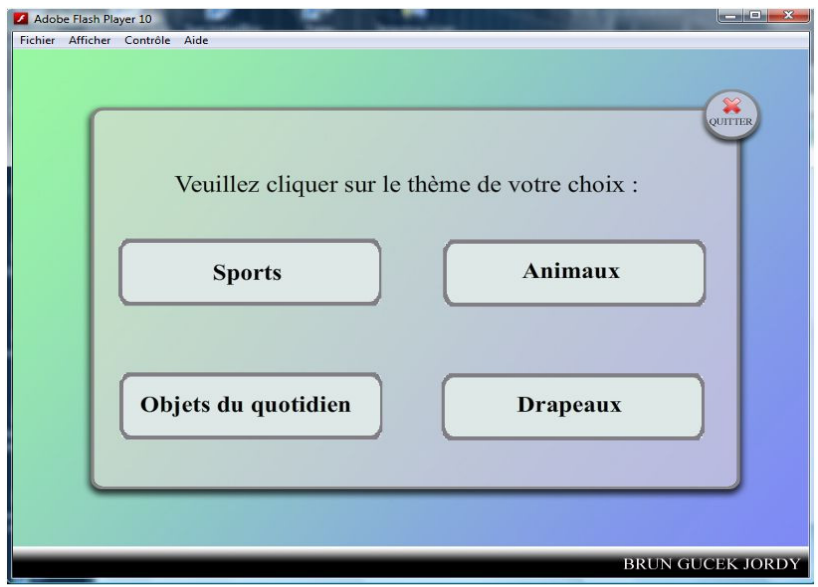

Fig. 2. First page of the parameters of the StudyTool Software 
This exercise of ProfessionalTool is a memory training exercise using slightly abstract figurative characters (hieroglyphics etc.). After memorizing these characters, they are displayed again but have to be recognized among other different pictures.

The second exercise of image memorization was designed for this study applying user centered design (see Fig.2). Initially, several adjustments were made to the memory training software program following well known ergonomic guidelines. To be able to guarantee the exercise usability both in substance and in form, we pretested a group of seniors. This approach enabled the final version of the exercise to be designed.

\subsection{User Tests}

In our user centered research we set about to test interaction as thoroughly as possible. We allowed for discussion time with the participants before each session to enable them to feel confident and able to ask any relevant questions, making sure that they could handle the tools, especially the mouse, particularly the participants with little practice.

The test period began with filling in the data sheet, followed by the test user and ending with the satisfaction questionnaire. This procedure was repeated for each software being studied.

Population Details. Our sample was made up of a total of $n=47$ participants. For the group of seniors the number of participants were $n=32$ the average age was 78,19 yrs old with $87,5 \%$ women and $12,5 \%$ men. As for the younger control group $n=15$ with an average age of 30,47 years old, $73,3 \%$ of whom were women and $26,7 \%$ men. $9,4 \%$ of the seniors had already used technology compared to $93,3 \%$ for the control group. The volunteers were either met in their homes or at a retirement home, which had displayed notices informing the residents of the study. The seniors were divided into 2 groups: A and B, group A began by testing ProfessionalTool, group B began with the StudyTool software. The groups formed were of the similar age and had the same level of computer skills.

Results. Only part of this study will be presented here. It deals with observation of the problems encountered. We compared the number of problems encountered, their frequency per minute, and finally the game time calculated per session (see Table1).

Regarding the number of problems encountered the feedback was very enlightening. For the senior group, the average number of problems is 8,63 lower for our version $(M=1,47 E D=1,46)$ than for ProfessionalTool's $(M=10,09 E D=4,30)$. The same tendency was found for the control group with a noticeably lower average for our version $(\mathrm{M}=, 60 \mathrm{ED}=, 74)$ than for ProfessionalTool 's $(\mathrm{M}=8,93 \mathrm{ED}=4,99)$. This result can be applied generally to the control group $(\mathrm{p}=, 000<, 05)$ as well as to the senior group $(\mathrm{p}=, 000<, 05)$. 
Table 1. Results of the assessment of problems of use ( Senior group )

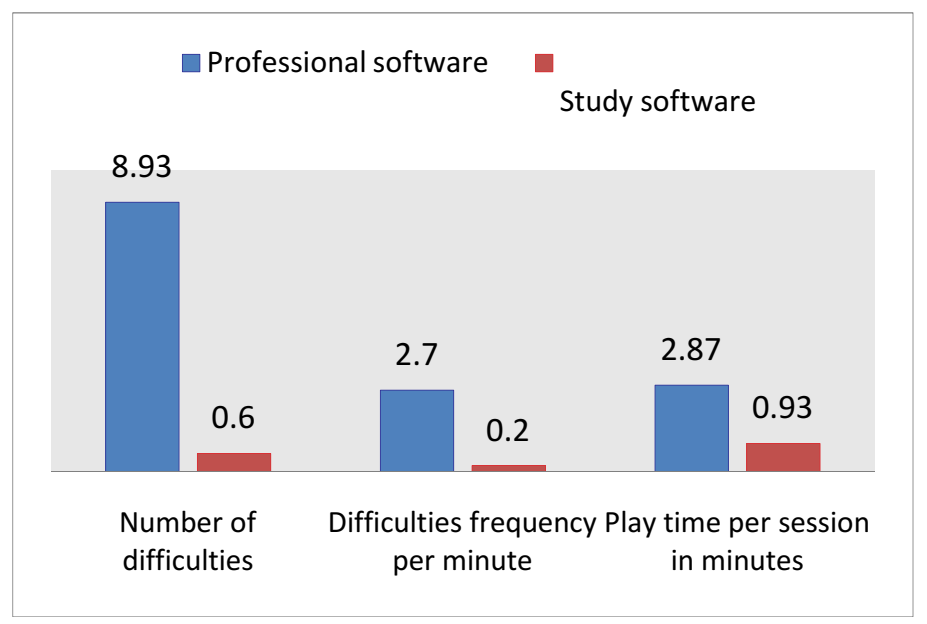

For the senior group the average frequency of the occurrence of problems is $(M=$ $, 00 \mathrm{ED}=, 000)$ for our version. It is significantly lower $(\mathrm{p}=, 000<, 05)$ than ProfessionalTool's $(\mathrm{M}=1,50 \mathrm{ED}=, 55)$. For the control group the average is also lower for our version $(M=, 20 \mathrm{ED}=1,63)$ than for ProfessionalTool' $(M=2,70 \mathrm{ED}=1,63)$. This result can be applied generally $(\mathrm{p}=, 000<, 05)$.

Significantly, for interaction time $(\mathrm{p}=, 000<, 05)$, the session times for our senior group, are clearly lower for our version $(\mathrm{M}=1,49 \mathrm{ED}=, 65)$ compared to ProfessionalTool's $(M=6,16 \mathrm{ED}=6,15)$. The situation is similar for the control group with a lower average time for our version $(\mathrm{M}=, 93 \mathrm{ED}=, 17)$ than for ProfessionalTool 's $(\mathrm{M}=2,87 \mathrm{ED}=1,36)$. This tendency can also be applied generally $(\mathrm{p}=, 000<, 05)$.

This tendency also appears for the control group with a much lower average for our version $(M=, 60 \mathrm{ED}=, 74)$ than for ProfessionalTool's $(\mathrm{M}=8,93 \mathrm{ED}=4,99)$.

The results of the satisfaction questionnaire also indicate problems concerning visual ergonomics guidelines, workload, control and error handling, uniformity and consistency, significance and compatibility. Taken as a whole the results of the evaluation eventually showed that ProfessionalTool has a low level of usability in terms of efficiency and the occurrence of user errors.

\section{Discussion}

This experience highlights the importance of certain ergonomic aspects of cognitive stimulation software. Their adaptation to a public with specific needs is often insufficient. This experience also underlines the necessity of the support of ergonomic recommendations (feed-back, usage intention, persuasion techniques engaging interaction, reinforcement...) and more generally, using an ergonomic approach to improve interface quality therefore enhancing use and acceptance of these tools. We must point out that these ergonomic improvements do not only benefit the population 
targeted, but also significantly benefit the control population; this is a typical result in research which is destined, in theory, to populations with specific needs.

\section{Conclusion}

Our study demonstrates the almost total lack of ergonomic considerations at the concept phase of the SC systems and to prove the positive effect of their inclusion. Beyond the actual effect of cognitive stimulation that is no longer in doubt, the challenge is to support the use and empower the user. This is only possible through tailored interactions..

According to $[12,13,14,15]$ these results give weight to the importance of the concept of "Perception of Gamification". This experience shows clearly that the lack of gamification implies a lower usage. Cognitive stimulation systems have to introduce persuasive technologies and graphic design to explain the interface's appearance and users commitment. To a certain degree, gamification will amplify the use of software. It would thus appear as a decisive factor for the design of a successful human-technology relationship beyond classic theories of technology adoption and use.

However, it relies on motivators dealing with nonfunctional needs; the usefulness of a system is not covered despite its importance, notably on work context. It thus questions the contribution of Gamification to casual systems, especially considering the kind of motivation triggered. We state that Gamification is about creating an interactive universe that would be simple, beautiful, appealing and engaging. It implies a will to mislead the user by modifying the core meaning of use: the conflicting relationship between elderly and cognitive stimulation.

Some Gamification upholders are currently talking about he concept of significant Gamification $[14,15,16]$, calling for user-centered game elements selection. It would be interesting to apply gamification criteria in order identify design rules that could be generalized in cognitive stimulation software.

Going beyond the scope of this research, we are currently working on a set of specific guidelines (intended for the designers of these systems) in which we incorporate knowledge from persuasion technology to enable enhanced acceptance and efficiency of these systems.

\section{References}

1. Study University of Louvain, http://www. uclouvain.be/cps/ucl/doc/.../ ML_article_chastel_veillissement.doc

2. World Health organization. Active aging: A policy framework. CITY: World Health Organization, (2002), http://whqlibdoc.who.int/hq/2002/WHO_NMH_NPH_02.8.pdf (September 1, 2011) (retrieved)

3. Lemaire, P.: La personne âgée, psychologie du vieillissement. De Boeck, Bruxelles (2005)

4. Auffray, C., Juhel, J.: Effets généraux et différentiels d'un programme d'entraînement cognitif multimodal chez la personne âgée. L'année Psychologique 101(1), 65-89 (2001) 
5. Büchel, F., Paour, J.L.: Déficience intellectuelle: déficit et rémédiation cognitive. Enfance 57(3), 227-240 (2005)

6. Hart, T.A.: Evaluation of websites for older adults: how 'senior-friendly' are they? Usability News 6(1) (February 2004), http: / / www. surl.org/usabilitynews / 61/older_adults.asp (accessed)

7. Kolodinsky, J., Cranwell, M., Rowe, E.: Bridging the generation gap across the digital divide: Teens teaching internet skills. Journal of Extension 40(3) (June 2002), http: / / www. joe.org/joe/2002 june/rb.html

8. Lewis, C.: HCI and cognitive disabilities. Interactions 13(3), 14-15 (2006)

9. Michel, G., Klein, M.: Utilisabilité et discrimination: étude préliminaire des machines à voter françaises. In: Actes de la Conférence IHM 2008. ACM Digital Library, Metz (2008)

10. http: / / www . w3 . org/WAI/

11. Bastien, J.M.C., Scapin, D.L.: A validation of ergonomic criteria for the evaluation of user interfaces. SIGCHI Bulletin 23, 54-55 (1991)

12. Marache-Francisco, C., Brangier, E.: Gamification experience: UXD with gamification background. In: Blashki, K., Isaias, P. (eds.) Emerging Research and Trends in Interactivity and the Human-Computer Interface, pp. 205-223. IGI-Global (2013)

13. Marache-Francisco, C., Brangier, E.: Perception of Gamification: between Graphical Design and Persuasive Design. In: Marcus, A. (ed.) DUXU 2013, Part II. LNCS, vol. 8013, pp. 558-567. Springer, Heidelberg (2013)

14. Marache-Francisco, C., Brangier, E.: Redefining Gamification. In: Blashi, K. (ed.) Proceedings of IADIS International Conference Interfaces and Human Computer Interaction 2012, pp. 227-231 (2012)

15. Marache-Francisco, C., Brangier, E.: Process For Gamification: From The Decision Of Gamification To Its Practical Implementation. In: The Sixth International Conference on Advances in Human oriented and Personalized Mechanisms, Technologies, and Services, CENTRIC 2013, Venice, Italy, October 27-November 1 (2013) ISSN: 2308-3492; ISBN: 978-1-61208-306-3

16. Duplàa, E., Kaufman, D., Sauvé, L., Renaud, L., Signalova, T., Emmanuel, T.: Méthode de spécifications de jeux sérieux adaptés aux ainés. Actes de l'atelier «Serious games, jeux épistémologique numériques ». In: EIAH 2013, pp. 14-18 (2013), http: / / eductice. ens-lyon.fr/EducTice/ressources/journees-scientifiques / atelierSG-EIAH2013/ActesatelierSG-EIAH2013 